\title{
Cristina Ricci
}

\section{L'eco letteraria del declino di Roma nel commento a Ezechiele di Girolamo e di Gregorio Magno}

Ascetismo e una certa ambiguità accomunano il "Rombild" di due Padri della Chiesa latina cronologicamente distanti tra loro, ma non privi di somiglianze: Girolamo e Gregorio Magno ${ }^{1}$. Rilevare come questi connotati si esprimano nei loro scritti è l'obiettivo di questo "viaggio intertestuale" dal primo al secondo, che si sofferma prima sul teologo stridonense ${ }^{2}$, poi - e più diffusamente, data l'ampiezza del brano sul tema in esame - sul vescovo romano ${ }^{3}$.

Tra i due si possono dunque cogliere alcune affinità: nelle loro biografie, ma soprattutto nei loro interessi: nel caso specifico, l'esegesi di Ezechiele . Tra l'altro costoro sono i soli, nella patristica latina, a dedicare a questo profeta un'opera intera: i corposi Commentarii in Hiezechielem di Girolamo e le Homiliae in Hiezechihelem (sic) di Gregorio, più esigue dei primi, ma anch'esse costituenti un insieme in sé compiuto, nonostante non presentino una spiegazione integrale del libro biblico.

Perché dunque entrambi gli autori trattano del declino di Roma proprio nell'esegesi a Ezechiele? Senz'altro per una coincidenza cronologica, riscontrabile nelle loro biografie: infatti, grosso modo nel periodo in cui sono impegnati a commentare questo libro, a Roma avvengono fatti drammatici, che li distolgono dalla meditazione sul testo biblico; eppure è proprio questo a fornire loro una chiave di lettura dei drammi di cui sono testimoni.

1 Per uno sguardo complessivo e storicamente contestualizzato sulle tappe della biografia e sull'opera dei due autori cf. Rebenich 2002, spec. 1-59 e la prima parte del saggio di Boesch Gajano 2004, spec. 15-147.

2 Sui testi geronimiani relativi al tema qui trattato (specie le lettere, ma talora anche il commentario a Ezechiele) cf. Courcelle 1964, 58-60; Paschoud 1967, 218-221; Zwierlein 1978, 49-53; Sugano 1983, 8. 54-64. 75. 119-122; Doignon 1990, 127 134; Inglebert 1996, 283-291, spec. 286-287; Feichtinger 1998, 151-154; Rebenich 2009, 52-59. Sul sacco di Roma del 410 e relative fonti cf. Heather 2005, $227-232$ e la recente pubblicazione di Ghilardi/Pilara 2010, 169-212.

3 Per la situazione di Roma ai tempi di Gregorio, la sua visione della città e il senso della fine ad essa connesso (specie in riferimento al brano di seguito analizzato), cf. Courcelle 1964, 257-258; Gregorovius 1988, 254-270; Krautheimer 1996, 75-102; Pietri 1991, 9-21; Markus 1997, 51-54.

4 Con Ezechiele in caratteri corsivi ci si riferisce al libro del profeta, in caratteri tondi (Ezechiele) alla persona. 
Il fatto che per entrambi i teologi Ezechiele sia stato oggetto di un'indagine approfondita non è sfuggito alla ricerca, specie di ambito gregoriano ${ }^{5}$; tuttavia le ragioni e le modalità in base a cui essi hanno messo in rapporto la profezia di Ezechiele coi disastri dei loro tempi non mi risultano ancora studiate in un'ottica comparativa.

Il presente contributo, quindi, propone una lettura di brani attinti per la maggior parte dai commenti di Girolamo e di Gregorio a Ezechiele, selezionati in base al loro esplicito legame col "Sitz im Leben" dei rispettivi autori. In questi testi si cerca di cogliere non solo affinità e differenze tra i due esegeti, ma anche risonanze di stilemi classici e biblici, che costoro rimodellano per forgiare il proprio "Rombild" e trasmettere la "Weltanschauung" ad esso connessa.

In via preliminare, va considerata la presenza di Roma nella biografia dei due autori: l'Vrbs è sede permanente per Gregorio (a parte il suo soggiorno costantinopolitano, dal 579 al 585 circa), invece per Girolamo lo è solo in via transitoria (dal 382 al 385). Nella città entrambi ricoprono ruoli importanti: quest'ultimo come "segretario" e consulente di papa Damaso, specie in ambito biblico-esegetico e nei rapporti con le chiese orientali ${ }^{6}$; Gregorio come praefectus urbi prima, poi come collaboratore di Pelagio II e suo apocrisario a Costantinopoli, infine - e fino alla sua morte - come vescovo di Roma (590-604). Entrambi sono dunque personaggi di spicco nel clero romano, ma anche d'eccezione a causa del loro stile di vita monastico: Girolamo, infatti, si propone come guida di vita ascetica per alcuni membri del patriziato, tra cui Paola ed Eustochio, che poi lo seguono nel 386 a Betlemme; Gregorio, esponente dell'aristocrazia senatoria, dopo aver ricoperto incarichi politici nella città, si ritira a più di trent'anni nel monastero da lui fondato sul Celio.

Per entrambi si può presumere una formazione tradizionale "romana", che deve aver inciso sulla loro percezione del ruolo dell'Vrbs nell'ecumene e nella storia. Anche per questo la crisi di Roma, culminante per Girolamo nel sacco dei Visigoti nel 410, per Gregorio nell'assedio dei Longobardi nel 593/594, li sconvolge, distogliendoli dalla loro concentrazione sull'attività esegetica.

A questa crisi, tuttavia, essi guardano da prospettive differenti: il primo da lontano, da Betlemme, l'altro, invece, da molto vicino: dal cuore stesso di Roma.

Nel suo monastero betlemita Girolamo sta per dedicarsi al commento di Ezechiele su richiesta della giovane discepola Eustochio, quando viene sorpreso

5 Per una panoramica sull'esegesi patristica di Ezechiele prima di Gregorio cf. Kessler 1995, 112-131. 241-244, spec. 242 sui rapporti tra il commento di Girolamo e quello di Gregorio, su cui cf. anche Schwank 1934, 42-51 (che purtroppo non mi è stato possibile consultare).

6 Cf. quanto attesta lo stesso Girolamo in Hier. epist. 45, 3 (ed. Hilberg, CSEL 54, 325, 8-9) ed epist. 123, 9 (ed. Hilberg, CSEL 56.1, 82, 14-16): in merito cf. Rebenich 1992, $143-153$. 
dalla notizia della morte di Marcella e Pammachio durante il sacco di Roma, che ha provocato non solo morti, ma anche numerosi fuggitivi. Alcuni, insieme a mendicanti e pellegrini, sono venuti ad affollare il monastero di Girolamo ${ }^{7}$ che, affaccendato nell'accoglierli, stenta a concentrarsi sulla spiegazione del libro di Ezechiele; tuttavia in pochi anni (tra fine $410 / 411$ e il 414$)^{8}$ riesce a completarla in 14 libri.

Incompleta, invece, rimane l'esegesi di Gregorio per ragioni da lui stesso addotte nella praefatio del II libro delle sue omelie su Ezechiele e nell'ultima di queste, composte tra la fine del 593 e l'inizio del $594^{9}$ : la causa principale è la marcia del re longobardo Agilulfo contro Roma che, insieme a una parte dell'Italia non ancora conquistata dai Longobardi, è soggetta al governo bizantino, ma di fatto abbandonata a se stessa. Agilulfo la assedia tra la fine del 593 e gli inizi del 594: è allora il vescovo della città a dover organizzarne la difesa, soccorrere le vittime, trattare coi nemici ${ }^{10}$. Per questo egli si trova costretto ad interrompere il ciclo di prediche su Ezechiele, tenute in quel periodo nella basilica del Laterano, forse durante la liturgia mattutina. Questa "Predigtreihe" pare intrapresa - come si desume ancora dalla prefazione al secondo libro - su richiesta di un gruppo di monaci, chierici, vescovi e membri della curia, che hanno pregato Gregorio di predicare proprio su Ezechiele ${ }^{11}$.

I motivi della scelta di questo libro profetico si possono forse evincere dal suo "Sitz im Leben": infatti anche Ezechiele ha profetizzato in tempi di crisi, tra i deportati in terra babilonese ${ }^{12}$. Il suo ruolo sacerdotale, sul quale si è innestato

7 Cf. la testimonianza in merito di Girolamo nelle prefazioni a Hier. in Ezech., lib. 3 e 7 (ed. Glorie, 91, 9-18 e 277, 3-22); cf. anche Stemberger 1987, 102.

8 Sulla datazione cf. l'introduzione all'edizione di Hier. in Ezech. (ed. Glorie, vii-ix); Fürst 2003, 117.

9 Cf. Greg. M. in Ezech. 2, praef. (ed. Adriaen, 205, 10-14); ibid., 11, 24 (ed. Adriaen, 397, 608-611). Altre allusioni a simili circostanze sono ibid. 1, 11, 6 e nella dedica delle omelie a Mariniano, vescovo di Ravenna (ed. Adriaen, 171, 103-110 e 3, 4-5). In generale sulle Homiliae in Hiezechihelem cf. Santi 2008, 163-166; in particolare sulla loro datazione cf. l'introduzione all'edizione di Greg. M. in Ezech. (ed. Adriaen, v); Kessler 1995, 72-75; Müller 2009, 253.

10 Sulle trattative coi Longobardi cf. l'allusione nell'undicesima omelia indicata nella nota precedente: Greg. M. in Ezech. 1, 11, 6 (ed. Adriaen, 171, 106-107) e Id. epist. 5, 39 (ed. Norberg, CChr.SL 140, 316, 66-69) sul pagamento di un tributo in cambio della pace coi Langobardi. Su Agilulfo e le circostanze dell'assedio cf. Kessler 1995, 64-70; Markus 1997, 102-104; Müller 2009, 282-285.

11 Cf. Greg. M. in Ezech. 2, praef. (ed. Adriaen, 205, 3). Sul luogo della predicazione cf. la testimonianza di due codici riportata da Adriaen nell'introduzione a Greg. M. in Ezech. (ed. Adriaen, v n. 1). Sul luogo e sul pubblico cf. Manselli 1983, 943-944; Kessler 1995, 92-96. 99-105; Müller 2009, 254-256.

12 Cf. Pohlmann 2008, 20-21 e 15-20 sul contesto storico del libro di Ezechiele; cf. anche Kessler 1995, 106-109. 
il compito di annunciare la Parola divina ricevuta in visione e "in pasto"13, lo avvicina ai suoi due interpreti, specialmente a Gregorio che, in quanto episcopus, è incaricato di fare anche lui, come il profeta, da speculator ${ }^{14}$, sollecito nel vigilare sul suo popolo oppresso dagli invasori. Analogamente a Ezechiele, che dopo il primo assedio di Nabucodonosor a Gerusalemme nel 597 ha annunciato altri attacchi stranieri come punizione per i peccati di Israele, anche i suoi esegeti vedono ora, negli assalti dei barbari, una punizione per i peccati dei Romani ${ }^{15}$. D'altronde, sempre prendendo spunto da Ezechiele, già Ambrogio ha scorto un nesso tra le profezie su Gog e le catastrofi provocate dai Goti nei territori dell'impero romano.

Ezechiele, dunque, trovandosi in esilio coi suoi compatrioti, ha ricevuto da Dio l'incarico di rivelare loro la volontà divina celata nelle angustie presenti e di illustrare la visione di un nuovo tempio a Gerusalemme ${ }^{16}$ : questo racconto, foriero di speranza e intriso di misteri, deve aver destato un interesse peculiare nel pubblico di Gregorio, tanto da indurre il vescovo, costretto a sospendere al quarto capitolo la predicazione su Ezechiele, ad aggiungere l'esegesi almeno di tale visione, saltando direttamente a $E z \cdot 40^{17}$. Ci si può chiedere, tuttavia, perché egli non abbia poi integrato la lacuna lasciata tra i primi quattro capitoli e il quarantesimo nemmeno quando, otto anni dopo $(601 / 602)^{18}$, ha sottoposto le sue omelie a revisione, per pubblicarle in due libri (rispettivamente con dodici omelie su $E z .1,1-4,3$ e dieci su Ez. 40,1-47): forse perché - azzardo un'ipotesi - quei capitoli sostanzialmente gli sembravano sufficienti per far capire l'attualità del messaggio di Ezechiele per sé e per i suoi contemporanei.

Qual è dunque l'incidenza della profezia biblica sulla visione di Roma sviluppata da Gregorio? E su quella di Girolamo?

13 Cf. Ez. 2, $8-3,3$.

14 Sull'incarico di speculator affidato a Ezechiele cf. Ez. 3, 17 e 33, 7; sul suo rapporto con la funzione episcopale nella percezione di Gregorio cf. Greg. M. in Ezech. 1, 11, 4-7 (ed. Adriaen, 170-172). Sull'argomento cf. Mohrmann 1977, 238-252; con specifico riferimento a Gregorio cf. Kessler 1995, 237-240; Ricci 2003, 188-190.

15 Cf. Ambr. fid. 2, 16, 137-138 (ed. Faller, 104-106, spec. 104, 5-7): Namque et futuram nostri depopulationem et bella Gothorum Ezechiel illo iam tempore profetauit ("Infatti Ezechiele già a quel tempo profetizzò sia la futura devastazione, da noi subita, sia le guerre dei Goti” - trad. dell'autrice). Sul tema cf. Luiselli 1997, 22-23; Ricci 2002, $185-193$, spec. $185-187$.

16 Cf. Ez. $40-48$.

17 Cf. Greg. M. in Ezech. 2, praef. (ed. Adriaen, 205, 1-6).

18 Ibid., praef. $(3,5-8)$. 


\section{Girolamo}

Marginale e poco evidente è il ruolo della profezia nel primo testo che incontriamo in questo "viaggio intertestuale" tra i due autori: il prologo dei Commentarii in Hiezechielem.

Girolamo, dedicandoli a Eustochio, inquadra anche il loro contesto storico e letterario: questo su Ezechiele è uno della serie dei suoi trattati sui profeti, situato tra Isaia e Geremia ${ }^{19}$; non è dunque un'opera isolata, composta ad hoc per una certa occasione, a differenza delle Homiliae in Hiezechihelem, che sono il solo scritto gregoriano dedicato a un libro profetico; però come queste è intrapreso in un momento particolarmente inquietante. $\mathrm{Al}$ monaco betlemita, infatti, sono appena pervenute notizie sconvolgenti: il sacco di Roma e la morte di nobili amici, tra cui Pammachio e Marcella, parente di Eustochio ${ }^{20}$.

Ed ecco la reazione di Girolamo:

Atque ita consternatus obstupui, ut nibil aliud diebus ac noctibus nisi de salute omnium cogitarem meque in captiuitate sanctorum putarem esse captiuum, nec possem prius ora reserare, nisi aliquid certius discerem dum inter spem et desperationem sollicitus pendeo aliorumque malis me crucio ${ }^{21}$.

E sono rimasto così attonito e costernato, da non aver altro pensiero di giorno e di notte se non la salvezza di tutti, e da sentirmi prigioniero per la prigionia dei santi e non poter aprir bocca prima di non aver appreso qualcosa di più certo, mentre oscillo, turbato, tra speranza e disperazione, e mi tormento per i mali degli altri. (trad. dell'autrice)

Parole di sgomento, dettate da empatia verso gli amici romani colpiti dalla sciagura: al modo paolino egli li chiama sancti e si mostra emotivamente solidale con loro ${ }^{22}$. A dettare questa espressione di empatia, però, non è solo l'affetto di un amico e una guida spirituale, bensì forse anche l'influsso del modello profetico che Girolamo ha sott'occhio: pure Ezechiele, infatti, ha dichiarato di essere in medio captiuorum $(E z .1,1)$, prigioniero tra prigionieri, e di dover restare muto finché Dio non gli avrebbe riaperto la bocca per profetizzare $\left(E z .3,26^{23}\right)$. Più tardi anche Gregorio ammetterà di non riuscire più a predicare

19 Cf. Hier. in Ezech., prol. (ed. Glorie, 3-4, 1-5. 30-31).

20 Ibid. (3, 5-7). Su Eustochio, sua madre Paula e su Marcella cf. Feichtinger 1995, $168-$ 188 e 209-212, inoltre anche su Pammachio cf. PLRE 1, 312. 542-543. 663. 674675; Fürst 2003, 174. 190-191. 199-200; in particolare sul loro rapporto con Girolamo cf. Rebenich 1992, 154-180 passim., 184-185 e 193-203 passim.

21 Hier. in Ezech., prol. (ed. Glorie, 3, 7-12).

22 Per l'appellativo sancti cf. 1Cor. 1, 1; Eph. 1, 1 ecc.; per l'espressione di solidarietà cf. l'eco di 1Cor. 9, 19-22.

23 Ez. 3, 26: et linguam tuam adherescere faciam palato tuo et eris mutus nec quasi uir obiurgans quia domus exasperans est; cf. anche Ez. 24, 27. 
per l'angustia dell'assedio ${ }^{24}$. Se riguardo a questo ammutolimento, oltre alle comprensibili motivazioni psicologiche, si volesse attribuire ai due esegeti una consapevole allusione a Ezechiele, l'impedimento nel parlare si arricchirebbe, paradossalmente, di una valenza profetica: infatti, come è stato osservato in merito a $E z .24$ sulla presa di Gerusalemme, anche il mutismo imposto da Dio al suo araldo diventa una testimonianza "passiva", ex silentio, del dramma da costui presagito e poi effettivamente avvenuto ${ }^{25}$.

Una simile reazione di ammutolimento è formulata da Girolamo con un'eco di Ez. 3, 26 (et linguam tuam adherescere faciam palato tuo) e forse anche di un verso senecano (sed haeret ore prima uox) ${ }^{26}$ subito prima della sua sentenza sul disastro del 410, espressa un paio d'anni dopo (413) alla vergine romana Principia e sigillata con una solenne clausola:

Haeret uox et singultus intercipiunt uerba dictantis. Capitur urbs, quae totum cepit orbem ${ }^{27}$.

La voce mi nuore in gola e i singhiozzi interrompono le parole, mentre detto: La città che aveva conquistato l'universo intero, cade. (trad. Cola, 1963, 280)

Analoga sentenza, ma più articolata e densa di pathos, è nel prologo a Ezechiele:

Postquam uero clarissimum terrarum omnium lumen exstinctum est, immo Romani imperii truncatum caput et, ut uerius dicam, in una urbe totus orbis interiit, "obmutui..." 28

Infatti, dopo che si è estinta la luce più chiara di tutte le terre o, piuttosto, è stato troncato il capo dell'impero romano, e, per dir meglio, in un'unica città è venuto meno tutto il mondo, "Sono rimasto muto ...". (trad. dell'autrice)

In questa climax metaforica tripartita Girolamo condensa la sua visione del ruolo di Roma e il relativo dramma: la fine del mondo nella caduta di Roma. Tale nesso causale, evidenziato pure nelle omelie gregoriane su Ezechiele $^{29}$, è

24 Greg. M. in Ezech. 2, 10, 24: Iam cogor linguam ab expositione retinere.

25 Per il profeta come "araldo" cf. la definizione di "profeta" in Aug. quaest. in hept. 2, 17 (ed. Fraipont, 76, 216-217): enuntiator uerborum Dei hominibus, qui Deum uel non possunt uel non merentur audire. Per la temporanea mancanza di parola di Ezechiele cf. Vogt 1981, 92-106, spec. 100-101; Pohlmann 2008, 187-191, spec. 188.

26 Cf. Sen. Oed. 1009. Hagendahl 1958, 257 rileva un passo simile in Hier. epist. 130, 5 (ed. Hilberg, CSEL 56.1, 180, 20): haesit nox faucibus, rinviante a Verg. Aen. 2, 774 o 3,48 .

27 Hier. epist. 127, 12 (ed. Hilberg, CSEL 56.1, 154, 16-17): forse un'eco del detto oraziano: Graecia capta ferum uictorem cepit (Hor. epist. 2, 1, 156) e di quello lucaneo: toto populi qui nascimur orbe ... urbs nos una capit (Lucan. 7, 400-402), riecheggiati per rappresentare una situazione storica pressoché opposta? Nessun accenno a ciò, tuttavia, nella menzione di questo passo geronimiano in Hagendahl 1958, 302. Su Principia cf. PLRE 2, 904; Feichtinger 1995, 215-217; Fürst 2003, 208.

28 Hier. in Ezech., prol. (ed. Glorie, 3, 12-15).

29 Cf. Greg. M. in Ezech. 2, 6, 24 (ed. Adriaen, 313, 598-601): cf. infra, testo e n. 81. 
condensato con lugubre efficacia nell'immagine allitterante del clarissimum terrarum omnium lumen extinctum, che proprio nella metafora del lumen rievoca i presagi di Lattanzio sulla fine dell'Vrbs, allora scongiurati ma ora, ai tempi di Girolamo, avveratisi ${ }^{30}$. La metaforica di Roma slitta poi dall'ambito "astronomico" a quello "anatomico" con la macabra immagine del caput truncatum forse una vaga suggestione del Pompeo di Lucano o di altre analoghe scene di morte $^{31}$ ? - poi col nesso paronomastico urbe / orbis, per il quale Roma trascina con sé il mondo, in un modo volutamente non precisato dalla vaghezza della metafora, nel suo fatale epilogo (interiit) ${ }^{32}$.

Questo "epitaffio" di Roma ha un'immediata ripercussione sul suo autore: infatti il suo andamento ipotattico si appoggia sul predicato obmutui, con cui Girolamo applica a se stesso la pericope di Ps. 38, 3-4 per ribadire, citandola insieme a Eccli. 22, 6, l'effetto di sbigottito ammutolimento provocatogli dal sacco del 410:

"obmutui et humiliatus sum et silui de bonis, et dolor meus renouatus est; concaluit intra me cor meum, et in meditatione mea exardescet ignis" (Ps. 38, 4), nec putaui illam sententiam neglegendam: "Musica in luctu importuna narratio" (Eccli. 22, 6) ${ }^{33}$.

"Sono rimasto muto e umiliato e ho taciuto privo di bene, e il mio dolore si è rinnovato; il mio cuore si è infiammato dentro di me, e nel mio pensiero divampa il fuoco" (Ps. 38,4), e non ho ritenuto da trascurare quella sentenza: "Come musica durante il lutto è un discorso inopportuno". (trad. dell'autrice)

Egli perciò ritiene inopportuno esporre in circostanze così luttuose il libro di Ezechiele, ma poi vi si applica per l'insistenza di Eustochio ${ }^{34}$, nonostante le varie interruzioni causate dai trambusti nel suo monastero preso di mira da folle di fuggiaschi, come lui stesso sottolinea nelle prefazioni al terzo e al settimo libro del suo commentario ${ }^{35}$.

30 Cf. Lact. diu. inst. 7, 25, 8 (ed. Brandt, 665, 4-5): lumen illud ... cuius interitu mundus ipse lapsurus est; su questa metafora riferita a Roma e sul suo contesto cf. Freund 2009, 578.

31 Cf. Lucan. 8, 711: una nota est Magno capitis iactura reuulsi. Cf. anche l'immagine di Orfeo decapitato in Verg. georg. 4, 523: caput a ceruice reuulsum. L'espressione geronimiana caput truncatum evoca comunque varie suggestioni letterarie, per le quali si rinvia a Consolino 2009, 169-171 (tuttavia non in riferimento al passo di Girolamo. Ringrazio comunque la dott.ssa Petra Schierl per avermi suggerito questo spunto di comparazione). Le reminiscenze di Virgilio e Lucano nel commentario a Ezechiele sono documentate da Hagendahl 1958, 240-244.

32 Anche qui si può cogliere un'eco, in senso inverso, del verso ovidiano: ingens orbis in Vrbe fuit (Ov. ars 1, 174); con ciò non si intende però affermare che tale allusione rovesciata fosse già nelle intenzioni di Girolamo.

33 Hier. in Ezech., prol. (ed. Glorie, 3, 15-18).

34 Cf. ibid. (3, 18-19. 24) e ibid. 3, praef. (91, 18-20).

35 Ibid. $(91,13-18)$ e ibid. 7 , praef. $(2-19)$. 
Nella prima di queste egli riprende la personificazione di Roma e la fonda su principi generali, di ascendenza classica:

Vera sententia est: "Omnia quae orta occidunt, et aucta senescunt" ${ }^{\text {"36 }}$. Et alibi: "Nihil est enim opere et manu factum, quod non conficiat et consumat uetustas ${ }^{337}$.

Vera è la sentenza: "Tutto quanto è nato muore, e quanto è cresciuto invecchia". E altrove: "Non vi è, infatti, nulla di quanto è fatto da mano d'uomo, che la vecchiaia non consumi e distrugga". (trad. dell'autrice)

Le due citazioni sono attinte rispettivamente dal discorso di Sallustio sulla deperibilità dei beni materiali e del corpo rispetto all'immortalità dell'anima e dalla lode fatta da Cicerone a Cesare, che aveva richiamato Marcello dall'esilio con un atto di clementia, il cui ricordo, a differenza di tanti trofei di guerra, avrebbe superato il logorio del tempo. Girolamo, nel riprendere verbatim queste dichiarazioni, isolandole dal loro contesto originario, ne fa delle massime di valore paradigmatico, con cui inserire la sua visione sul destino di Roma nello schema biologico d'interpretazione della storia: l'antica "Lebensaltermetapher" ${ }^{\prime 8}$, che poi riprenderà anche Gregorio, ma sulla base di modelli non più classici, bensì biblici ${ }^{39}$. Per entrambi vale per Roma il processo evolutivo cosmico, secondo cui la città che ha assoggettato il mondo è anch'essa soggetta alla consunzione del tempo. Di suggestione classica e insieme tragica è l'antitesi usata dallo Stridonense per esprimere ciò:

Quis crederet ut totius orbis exstructa uictoriis Roma corrueret, ut ipsa suis populis et mater fieret et sepulcrum ... ? ?0 $^{40}$

Chi avrebbe creduto che Roma, edificata grazie alle vittorie su tutto il mondo, crollasse, che essa stessa diventasse per i suoi popoli madre e sepolcro ... ? (trad. dell'autrice)

36 Sall. Iug. 2, 3 (ed. Kurfess, 54, 7-8) ha ortaque; -que ovvero quae (preferito da Glorie) è omesso nell'edizione maurina e di Vallarsi (cf. PL 25, 75D e l'edizione Glorie, 91, apparat. crit. ad 1. 5). Sul ricorso a Sallustio da parte di Girolamo cf. Hagendahl 1958, 239. 292-294. La stessa citazione si trova in un noto brano di Cipriano sulla senectus mundi: Cypr. Demetr. 3 (ed. Simonetti, 36-37, 64-65; in merito cf. Zocca 1995, 645646): Haec sententia mundo data est, haec Dei lex est ut omnia orta occidant et aucta senescant. Forse Girolamo conosce questo precedente, dato che, come Cipriano, pare considerare la citazione sallustiana una sententia di valore universale.

37 Hier. in Ezech. 3, praef. (ed. Glorie, 91, 4-7).

38 Il tema della senectus mundi e lo schema biologico di periodizzazione della storia, diffusi in ambito sia pagano, sia cristiano, sono oggetto di numerosi studi: cf. ad es. Schmidt 1956, 288-317; Häussler 1964, 313-341; Luneau 1964, 53-78; Demandt 1978, 37 45; Siniscalco 1983, 37-47; Zocca 1995, 641-677.

39 Ovvero l'aquila del profeta Michea: s. infra, testo e note 76 e 77.

40 Hier. in Ezech. 3, praef. (ed. Glorie, 91, 7-9). 
Omniparens e sepulcrum è definita da Lucrezio la terra ${ }^{41}$ e, se Roma ne è signora, trasferirle questi attributi è senz'altro legittimo per Girolamo, che riconosce alla capitale dell'impero la funzione non solo di dominarlo, ma anche di provvedervi con premure "materne". Un analogo, stridente contrasto si ritrova nella lettera alla giovane patrizia Demetriade (414), fuggita dalla sua città a Cartagine e lodata dall'asceta per la sua scelta di rinuncia alle nozze, tanto più raccomandabile in un mondo in rovina:

Vrbs tua, quondam orbis caput, Romani populi sepulchrum est ${ }^{42}$.

La tua città, un tempo capitale del mondo, è ora la tomba del popolo romano. (trad. Cola 1963, 4, 310)

Tuttavia questo dominio - lamenta l'esegeta di nuovo nel commento a Ezechiele - si è ormai ridotto in miseria:

... ut tota Orientis, Aegypti, Africae littora olim dominatricis urbis seruorum et ancillarum numero complerentur: ut cotidie sancta Bethleem, nobiles quondam utriusque sexus atque omnibus diuitiis affluentes, susciperet mendicantes? ?3 $^{43}$

[chi lo avrebbe creduto] che tutte le coste dell'Oriente, dell'Egitto, dell'Africa si riempissero di una quantità di schiavi e schiave della città un tempo dominatrice, che la santa Betlemme accogliesse ogni giorno come mendicanti persone di entrambi i sessi, un tempo nobili e abbondanti d'ogni ricchezza? (trad. dell'autrice)

Rappresentando così la folla in fuga dalla città, Girolamo evidenzia per Roma un capovolgimento di sorti: dall'antica potenza all'attuale debolezza, dall'abbondanza di beni e di sudditi alla povertà e allo spopolamento, che sono conseguenze degli attacchi dei barbari. La cosiddetta olim dominatrix urbs si è dunque trasformata in un luogo di morte, dal quale fuggire.

\section{Gregorio}

Scenari affini sono dipinti da Gregorio in un inquietante excursus su Roma, definito da Ferdinand Gregorovius "Leichenrede am Grabe Roms" ${ }^{44}$.

L'autore inserisce questa "orazione funebre" dopo la spiegazione allegoricomorale di $E z .40,19$ riguardante alcune misure del tempio visitato dal profeta nella sua visione. Egli interpreta la distanza tra una porta e un atrio interno come la contemplazione, mentre la porta sarebbe la fede che dà accesso alla

41 Lucr. 5, 259: omniparens eadem rerum commune sepulcrum.

42 Hier. epist. 130, 5 (ed. Hilberg, CSEL 56.1, 180, 4). Su Demetriade cf. PLRE 2, $351-$ 352; Feichtinger 1995, 217-220; Fürst 2003, 168-169.

43 Hier. in Ezech. 3, praef. (ed. Glorie, 91, 9-13): il punto interrogativo conclude la domanda iniziata con Quis crederet (91, 7: v. supra, testo e n. 40).

44 Gregorovius 1988, 259. 
conoscenza di Dio e si manifesta nella rettitudine morale e nella carità di chi rinuncia ai beni materiali, senza desiderare in cambio alcuna gloria terrena ${ }^{45}$.

Affinché questa lettura in senso etico-spirituale, proposta ad ascoltatori particolarmente sensibili alla gravità delle circostanze, possa servire da ammonimento all'ascesi e all'impegno per il bene, il predicatore le conferisce forza persuasiva col metterla in rapporto con la drammaticità del presente da loro vissuto. Egli infatti, appellandosi ai suoi uditori con l'apostrofe fratres $m e i^{-46}$, fa loro constatare lo scenario desolante che li circonda:

Ipsa autem quae aliquando mundi domina esse uidebatur qualis remanserit Roma conspicitis. Immensis doloribus multipliciter attrita, desolatione ciuium, impressione hostium, frequentia ruinarum ${ }^{47}$.

Vedete com'è ridotta Roma stessa, che un tempo sembrava la dominatrice del mondo. Schiacciata in tanti modi da immensi dolori, dalla desolazione dei cittadini, dall'attacco dei nemici, dalle continue rovine. (trad. Gandolfo 1993, 171 con modifiche dell'autrice)

L'epiteto mundi domina si ritrova identico in un predecessore di Gregorio, Leone Magno ${ }^{48}$, ma, cambiati i tempi, Gregorio vi aggiunge aliquando ${ }^{49} \mathrm{e}$ uidebatur, lasciando intendere il carattere apparente ed effimero dell'antica dominatio che, sotto i colpi dei Longobardi, si è trasformata in desolatio.

"Desolazione" è un concetto ricorrente nelle profezie di Ezechiele ${ }^{50}$, e l'esegeta lo usa come cerniera per saldare il suo "Rombild" alla profezia di $E z .24$ su Gerusalemme - ma lui dice Samaria, forse confondendosi con Ez. 23, relativo appunto alla punizione di Samaria, anch'essa infedele a Dio come Gerusalemme. Il passo sopra citato, infatti, dopo la menzione di desolazione e oppressione, sofferenze e rovine, prosegue così:

ita ut in ea [sc. Roma] completum esse uideamus quod contra urbem Samariam per hunc eundem prophetam longe superius dicitur: "Pone ollam, pone, inquam, et mitte in ea aquam, et congere frusta eius in ea." Et paulo post: "Efferbuit coctio eius, et discocta sunt ossa illius in medio eius." Atque iterum: "Congere ossa, quae igne succendam: consumentur carnes, et coquetur uniuersa compositio, et ossa tabescent. Pone quoque eam super prunas uacuam, ut incalescat, et liquefiat aes eius." (Ez. 24, 3-5.10-11) ${ }^{51}$.

45 Greg. M. in Ezech. 2, 6, 16 e ibid., 19 (ed. Adriaen, 307. 309, 394-398 e 467-475).

46 Ibid., 22 (311, 533).

47 Ibid. $(311,534-537)$.

48 Leo M. tract. 82 (ed. Chavasse, 513, 95). L'appellativo equivalente di orbis domina, apposto a Roma, si trova ad es. in Hier. in Is. 16, 57, 7-8 (ed. Adriaen, 646, 12); Pelag. epist. ad Demetr. 30 (PL 30, 44AB).

49 Analogamente Girolamo definisce Roma olim dominatrix (Hier. in Ezech. 3, praef., ed. Glorie, 91, 9-10).

50 Cf. p. es. Ez. 6, 14;12, 19-20;14, 16;15, 8; $19,7$.

51 Greg. M. in Ezech. 2, 6, 22 (ed. Adriaen, 311, 537-545). 
in essa vediamo adempiuto ciò che tanto tempo prima questo medesimo profeta vaticinò contro la città di Samaria: "Metti su la pentola, dico, versaci acqua e mettici dentro i pezzi di carne." E poco dopo: "E falla bollire molto, sì che si cuociano dentro le ossa." E ancora: "Raccogli insieme le ossa perché brucino; fa' consumare la carne, riducila in poltiglia e le ossa scompaiano. Metti anche la pentola vuota sulla brace, prchè si arroventi e il rame si sciolga”. (trad. Gandolfo 1993, 171)

Nel brano di $E z .24,3-11$ si descrive un processo di cottura il cui valore allegorico è già previsto dal sensus auctoris ${ }^{52}$, ovvero da Ezechiele e da chi lo ispira, e rinvia all'assedio posto da Nabucodonosor a Gerusalemme nel 588/587 a.C., preannunciato dal profeta ad ulteriore aggravio delle angustie dell'esilio, nel quale lui e il suo popolo già si trovavano.

Nel suo commentario, Girolamo ha spiegato questa allegoria profetica in senso storico e morale, per poi riferirla alle vicende dell'assedio e incendio di Gerusalemme rispettivamente sotto Tito e Adriano e infine, in prospettiva escatologica, al giudizio finale $\mathrm{e}^{53}$.

Diversa, invece, e molto originale è l'interpretazione di Gregorio, che stabilisce un parallelo tra ogni fase della cottura esposta dal profeta e la storia di Roma:

Tunc enim nobis olla posita est, cum haec est ciuitas constituta. Tunc in ea aqua missa est et frusta eius congesta sunt, quando ad eam undique populi confluebant, qui uelut aqua calens actionibus mundi feruescerent et quasi frusta carnium in ipso suo feruore liquarentur. De qua bene dicitur: "Efferbuit coctio eius, et discocta sunt ossa illius in medio illius" (Ez. 24, 5), quia prius quidem in ea uehementer incaluit actio gloriae saecularis, sed postmodum ipsa gloria cum suis sequacibus defecit ${ }^{4}$.

Allora è stata messa su la pentola per noi, quando è stata costruita questa città. Allora è stata versata in essa l'acqua e vi sono stati messi i pezzi di carne, allorché da ogni parte confluivano in essa i popoli, che in certo modo si scaldavano e bollivano come acqua per le imprese del mondo e nel loro stesso calore si scioglievano come pezzi di carne. Di essa è detto bene: "E falla bollire molto, sì che si cuociano dentro anche le ossa", poiché dapprima in essa si riscaldò in modo veemente l'attività per la gloria temporale, ma poi questa gloria è venuta meno con quelli che la perseguivano. (trad. Gandolfo 1993, 171-173)

La messa sul fuoco della pentola corrisponde, dunque, alla fondazione della città, nella quale sono poi confluite diverse genti, che s'infervoravano e si consumavano negli affari di questo mondo, come l'acqua che bolle coi pezzi di carne.

La metafora del ribollire dell'actio gloriae saecularis non è priva di ambiguità, poiché allude a un fervore di attività che implica in sé una dinamica di autoconsunzione, desumibile dal parallelismo tra il liquefarsi di quanto viene cotto e

52 Cf. Ez. 24, 3: et dices per prouerbium ad domum inritatricem parabolam.

53 Cf. Hier. in Ezech. 7, 24, 1-14 (ed. Glorie, 326, 1393-1403).

54 Greg. M. in Ezech. 2, 6, 22 (ed. Adriaen, 311, 545-553). 
il dileguarsi dell'antica gloria romana col venire meno dei suoi sostenitori. Questo è il giudizio retrospettivo di Gregorio, che in effetti, a un certo punto della sua carriera politica, ha lasciato i negotia secolari per l'otium contemplativo del monastero ${ }^{55}$.

L'allegoresi continua poi nei dettagli:

Per ossa etenim potentes saeculi, per carnes uero populi designantur, quia sicut carnes portantur ossibus, ita per potentes saeculi infirmitas regitur populorum ${ }^{56}$.

Le ossa infatti indicano i potenti del mondo, le carni invece i popoli, poiché come le carni sono sostenute dalle ossa, così la debolezza dei popoli è governata dai potenti del mondo. (trad. Gandolfo 1993, 173).

Nell'identificare le carni coi popoli e le ossa coi potenti e più avanti col senato, che reggono la compagine sociale ${ }^{57}$, l'autore rivela una visione della società a cui appartiene che è d'impronta indubbiamente aristocratica, ma è insieme critica, in quanto consapevole della transitorietà e del vizio insiti in tale struttura ${ }^{58}$ : infatti ceto dirigente e sudditi sono ormai venuti meno, come le ossa e le carni decomposte durante la cottura:

Sed ecce iam de illa omnes huius saeculi potentes ablati sunt; ossa ergo excocta sunt. Ecce populi defecerunt, carnes eius liquefactae sunt. Dicatur itaque: "Congere ossa, quae igne succendam; consumentur carnes, et coquetur uniuersa compositio eius, et ossa tabescent" (Ez. 24, 10) ${ }^{59}$.

Ma ecco che ormai da essa sono stati eliminati tutti i potenti del mondo; perciò sono state cotte le ossa. Ecco, i popoli sono venuti meno; le sue carni si sono sciolte. Perciò si dica: "Raccogli insieme le ossa, perché siano bruciate dal fuoco; si consumino le carni e si cuocia tutta la sua poltiglia e le ossa scompaiano". (trad. Gandolfo 1993, 173)

Il compimento della profezia nel presente trova conferma nelle domande retoriche che la seguono:

Vbi enim senatus? Vbi iam populus?

55 Negotia e otium / otia sono termini usati da Gregorio stesso in simili contesti: cf. Greg. M. in euang. 1, 17, 14 (ed. Étaix, 128, 299) per il primo e Id. epist. 1, 25 (ed. Norberg, CChr.SL 140, 33, 14-15) per il secondo: su questa terminologia cf. Leclercq 1963, 3641.

56 Greg. M. in Ezech. 2, 6, 22 (ed. Adriaen, 311, 553-556).

57 Anche Ezechiele riferisce metaforicamente le "carni" ai capi del popolo, come è evidente in Ez. 11,2-3. Sul senato all'epoca di Gregorio, da identificare con un ceto aristocratico dotato di poteri ormai ridotti rispetto a quelli del senato antico, cf. Arnaldi 1982, 6-13. $34-37$ con riferimento anche al passo dell'omelia qui sopra citato.

58 Cf. Greg. M. in Ezech. 2, 6, 22 (ed. Adriaen, 312-313, 574-576. 580-581. 586-587. 596-597, citati in seguito: testo e n. 68.72.75.77).

59 Ibid. $(311,556-560)$.

60 Ibid. $(311-312,560-561)$. 
Dov'è, infatti, il senato? Dov'è ormai il popolo? (trad. Gandolfo 1993, 173)

Sfogo amaro, autentico, benché non privo di precedenti letterari (domande analoghe, infatti, le ha poste Pelagio scrivendo sul sacco del $410^{61}$ ): è la voce del superstite, che vive l'apocalittico spopolarsi della sua città:

Quia enim senatus deest, populus interiit, et tamen in paucis qui sunt dolores et gemitus cotidie multiplicantur, iam uacua ardet Roma ${ }^{62}$.

Dato che manca il senato, il popolo è scomparso, e tuttavia nei pochi superstiti si moltiplicano ogni giorno dolori e gemiti, Roma, ormai vuota, brucia. (trad. Gandolfo 1993, 173, con modifiche dell'autrice)

In questa clausola di cadenzata gravità viene inscenato il dramma della città in fiamme, vuota perché, oltre ai morti, non pochi sono stati fatti prigionieri o sono fuggiti, un po' come nel $410^{63}$. Indipendentemente dal fatto che $\mathrm{i}$ Longobardi di Agilulfo abbiano messo a fuoco l'Vrbs o no, in questa scena dipinta da Gregorio si fondono varie suggestioni letterarie: certamente quella della città incendiata e desolata, piena solo di gemiti, che già nel profetismo biblico è la tipica stilizzazione della catastrofe sintomo di condanna ${ }^{64}$; inoltre forse pure quella di antichi oracoli sulla fine di Roma, vittima del fuoco ${ }^{65}$. Così anche Girolamo, in riferimento al sacco dei Visigoti, ha rappresentato il bruciare di Roma con un'enfasi probabilmente sproporzionata alla realtà dei fatti, ma suggerita da simili immagini apocalittiche ${ }^{66}$.

$\mathrm{Al}$ di là del realismo o meno dell'immagine di Roma incendiata, il senso del suo irreversibile declino si coglie anche nello sfacelo dei monumenti, paragonato da Gregorio al disfarsi della stessa pentola dopo il suo contenuto:

Quid autem ista de hominibus dicimus, cum, ruinis crebrescentibus, ipsa quoque eius destrui aedificia uidemus? Vnde apte de ciuitate iam uacua subditur: "Incalescat, et liquefiat aes eius" (Ez. 24, 11). Iam enim et ipsa olla consumitur, in qua prius et carnes et ossa consumebantur, quia postquam defecerunt homines, etiam parietes cadunt ${ }^{67}$.

61 Cf. Pelag. epist. ad Demetr. 30 (PL 30, 44B) : Vbi tunc nobilitatis ordo? Vbi certi et distincti illius dignitatis gradus?

62 Greg. M. in Ezech. 2, 6, 22 (ed. Adriaen, 312, 566-569).

63 Cf. quanto riferisce in merito Girolamo (per il pertinente rinvio v. supra, testo e n. 7).

64 Cf. ad es. Ez. 23, 25; Am. 1, 7. 10. 12. 14; 2, 2 ecc.

65 Cf. ad es. orac. Sibyll. 3, 52-54. Sul tema cf. Doignon 1990, 123-127.

66 Hier. epist. 128, 5 (ed. Hilberg, CSEL 56.1, 161, 9-12): Pro nefas, orbis terrarum ruit et in nobis peccata non corruunt. Vrbs inclita et Romani imperii caput uno hausta est incendio. Nulla regio, quae non exules eius habeat. In cineres ac fauillas sacrae quondam ecclesiae conciderunt ("Che schifo! L'universo intero sta crollando, ma i peccati in noi non accennano a crollare! La città illustre, la capitale dell'impero romano è stata distrutta da un solo incendio. Non c'è regione che non accolga i suoi profughi. Chiese un tempo consacrate al culto sono finite in cenere e polvere" - trad. Cola, 1963, 289).

67 Greg. M. in Ezech. 2, 6, 22 (ed. Adriaen, 312, 569-574). 
Ma perché diciamo ciò in riferimento agli uomini, dato che, col crescere delle rovine, vediamo distrutti gli stessi suoi edifici? Per cui della città ormai vuota si aggiunge a ragione: "Si riscaldi e si sciolga il suo bronzo". Infatti ormai si consuma la pentola stessa, nella quale prima si consumavano le ossa e le carni, poiché dopo che sono venuti meno gli uomini, cadono anche i muri. (trad. Gandolfo 1993, 173)

Per il vescovo romano, quindi, gli edifici sono parti integranti della città, come i suoi abitanti: scomparsi gli uni, fatiscenti gli altri, svanisce la gloria di Roma, che pare dileguarsi sotto l'incalzare di una serie martellante di domande retoriche, di nuovo introdotte da $V b i$ :

Vbi autem sunt qui in eius aliquando gloria laetabantur? Vbi eorum pompa? Vbi superbia? Vbi frequens et immoderatum gaudium? ${ }^{38}$

Dove sono dunque quelli che un tempo tripudiavano per la sua gloria? Dov'è il loro fasto? Dove la superbia? Dove l'allegria continua e smodata? (trad. Gandolfo 1993, 173 con modifiche dell'autrice)

Con queste interrogative l'autore risposta il suo punto d'osservazione dall'esterno, da cui nota la decadenza degli edifici di Roma, all'interno, ossia al vizio insito nella gloria romana, consistente nell'ostentazione, nella superbia e nella smoderatezza.

Per esprimere questo, Gregorio adduce un'altra allegoria profetica, che interpreta ancora in chiave attualizzante:

Impletum est in ea quod contra destructam Niniuen per prophetam dicitur: "Vbi est habitaculum leonum et pascua catulorum leonum?" (Nah. 2, 11) ${ }^{69}$.

Si è adempiuto in essa quanto il profeta dice contro Ninive distrutta: "Dov'è la tana dei leoni e il pascolo dei cuccioli dei leoni?" (trad. Gandolfo 1993, 173 con modifiche dell'autrice)

Qui Roma viene associata a Ninive, città punita con la distruzione, poiché entrambe paragonabili a "tane di leoni".

La figura del leone è presente anche in Ezechiele, ma in riferimento ad Israele che fa preda di nemici, ma viene catturato prima dagli Egiziani, poi dai Babilonesi ${ }^{70}$. Gregorio però preferisce attingere qui al profeta Nahum, forse perché nell'immagine dell'habitaculum leonum fa meglio trasparire le sue riserve sul ruolo di Roma come centro di potere secolare e di formazione per i rampolli dei potenti: la tana di leoni racchiude in sé, infatti, quei connotati di "Ehrgeiz" ", di superbia e d'intemperanza, come quelli denunciati poco prima dal predicatore. Infatti egli dichiara:

68 Ibid. $(312,574-576)$.

69 Ibid., $23(312,577-579)$.

70 Cf. Ez. $19,1-9$.

71 In questo contesto il termine "Ehrgeiz" mi sembra più efficace, a livello semantico, rispetto ad "ambizione". 
An eius duces ac principes leones non erant, qui, per diuersas mundi prouincias discurrentes, praedam saeuiendo et interficiendo rapiebant? Hic leonum catuli inueniebant pascua, quia pueri, adolescentes, iuuenes saeculares et saecularium filii huc undique concurrebant, cum proficere in hoc mundo uoluissent ${ }^{72}$.

Non erano forse leoni i suoi condottieri e capi, che, scorazzando attraverso le regioni del mondo, ghermivano la preda infierendo e uccidendo? Qui i cuccioli dei leoni trovavano pascolo, poiché fanciulli, adolescenti, giovani di questo secolo e i loro figli vi accorrevano da ogni dove, quando volevano far carriera in questo mondo. (trad. Gandolfo 1993, 173, con modifiche dell'autrice)

Gregorio non è il primo a connotare la politica espansionistica romana nel senso di una certa aggressività "predatoria"73, ma vi aggiunge una nota di effimero, di vanità, stagliandovi contro - con l'efficace avversativa "attualizzante" del sed ecce, già adottata a riguardo dello sgretolarsi della struttura sociale tradizionale ${ }^{74}-$ una realtà presente specularmente rovesciata: dall'affollamento di centro di potere e di cultura alla desolazione e decadenza culturale, dalla potenza all'oppressione, dall'allegria smodata ai gemiti di dolore:

Sed ecce iam desolata, ecce contrita, ecce gemitibus oppressa est. Iam nemo ad eam currit, ut in hoc mundo proficiat, iam nullus potens et uiolentus remansit, qui opprimendo praedam diripiat ${ }^{75}$.

Ma ecco ormai è desolata, eccola calpestata, eccola soffocata dai gemiti. Ormai nessuno vi accorre, per far carriera in questo mondo, ormai non è rimasto nessun potente e violento, che strappi la preda con l'oppressione. (trad. Gandolfo 1993, con modifiche dell'autrice)

Questa parabola storica è sintetizzata da Gregorio con l'allegoria dell'aquila del profeta Michea:

Contigit ei quod de Indaea nouimus per prophetam dictum: "Dilata caluitium tuum sicut aquila". (Mich. 1, 16)

Le è accaduto ciò che per mezzo del profeta sappiamo della Giudea: "Diventa calva come un'aquila”. (trad. Gandolfo 1993, 173)

così parafrasata:

Caluitium ergo suum sicut aquila dilatat, quia plumas perdidit, quae populum amisit. Alarum quoque pennae ceciderunt, cum quibus uolare ad praedam consueuerat, quia omnes eius potentes exstincti sunt, per quos aliena rapiebat ${ }^{7}$.

72 Greg. M. in Ezech. 2, 6, 23 (ed. Adriaen, 312, 579-584).

73 La constatazione dell'ascesa di Roma come causa di sofferenza per i vinti emerge in diversi autori cristiani: cf. ad es. Oros. hist. 5, 1, 13 (ed. Arnaud-Lindet, t. 2, 84-85); Salv., gub. 5, 9, 46 (ed. Lagarrigue, 346, 13-20).

74 Greg. M. in Ezech. 2, 6, 22 (ed. Adriaen, 311, 556: v. supra, testo e n. 59).

75 Ibid., 23 (312, 584-587). Cf. in merito Luiselli 1992, 726-727.

76 Greg. M. in Ezech. 2, 6, 23 (ed. Adriaen, 312, 588-590).

77 Ibid. (312-313, 593-597). 
Perciò Roma, che ha perduto il popolo, diventa sempre più calva come un'aquila che ha perduto le piume. Sono cadute anche le penne, con le quali era solita volare sulla preda, perché tutti i suoi potenti, per mano dei quali depredava i beni altrui, sono scomparsi. (trad. Gandolfo 1993, 173-175)

Si può supporre che sia stata la figura della "grande aquila dalle grandi ali e dalle lunghe penne, folta di piume” dipinta da Ezechiele $(E z .17,3)$ a suggerire all'esegeta, per contrasto, il rapace ormai spennacchiato di Michea, le cui penne e piume, come prima le ossa e la carne, sono ora interpretate come il ceto dirigente e il popolo che, diradatisi, hanno lasciato la città spoglia e decrepita: simbolo non più di eternità, di imperium sine fine ${ }^{78}$, ma di peribilità $\mathrm{e}$ decadenza. In questa rappresentazione dell'aquila, inoltre, convergono i simboli della Romanitas imperiale e gli indizi della vecchiaia a cui è soggetta ogni civiltà, secondo la "Lebensaltermetapher" qui solo sottintesa, ma più esplicita in altri testi gregoriani ${ }^{79}$, oltre che nella prefazione sopra commentata di Girolamo ${ }^{80}$.

Infatti Gregorio all'immagine dell'aquila di Roma aggancia la constatazione che il processo di decadenza dilaga ben oltre i confini dell'Vrbs:

Haec autem quae de Romanae urbis contritione dicimus, in cunctis facta mundi cinitatibus scimus. Alia etenim loca clade desolata sunt, alia gladio consumpta, alia fame cruciata, alia terrae hiatibus absorpta ${ }^{81}$.

Ma quanto diciamo della rovina di Roma, sappiamo che è avvenuto in tutte le città del mondo. Infatti alcuni luoghi sono stati desolati dalla strage, altri annientati dalla spada, altri tribolati dalla fame, altri inghiottiti dai terremoti. (trad. Gandolfo 1993, 175 con modifiche dell'autrice)

Vrbs ed orbis hanno dunque lo stesso destino: per Gregorio come per Girolamo che, in effetti, poco prima del 410 ha scritto: Quid saluum est, si Roma perit?, invertendo il detto encomiastico di Lucano: quid satis est, si Roma parum est? allo scopo di persuadere la giovane vedova Geruchia ${ }^{82}$ a non risposarsi in tempi così calamitosi.

Non molto diversa è la "Untergangsstimmung" che contraddistingue l'inizio dell'excursus gregoriano su Roma:

78 Cf. Verg. Aen. 1, 279.

79 Un esempio di rilievo, tra i numerosi, è l'incipit della prima omelia sui Vangeli: Greg. M. in euang. 1, 1, 1 (ed. Étaix, 5, 1-3). Cf. Bartelink 2000, 139-145, spec. 140-141.

80 Cf. Hier. in Ezech. 3, praef. (v. supra, testo e n. 36-37. 40).

81 Greg. M. in Ezech. 2, 6, 24 (ed. Adriaen, 313, 598-601).

82 Cf. Hier. epist. 123, 16 (ed. Hilberg, CSEL 56.1, 94, 3-6): Potentiam Romanae urbis ardens poeta describens ait: Quid satis est, si Roma parum est? (Lucan. 5, 274) Quod nos alio mutemus elogio: Quid saluum est, si Roma perit? Su Geruchia cf. PLRE 2, 509; Feichtinger 1995, 208-209; Fürst 2003, 180-181; inoltre, con particolare riferimento alla lettera a lei indirizzata cf. Rebenich 1992, 285-289. 
Quid est iam, rogo, quod in hoc mundo libeat? Vbique luctus aspicimus, undique gemitus audimus. Destructae urbes, euersa sunt castra, depopulati agri, in solitudine terra redacta est ${ }^{83}$.

Che c'è ormai, domando, che ci attiri in questo mondo? Dovunque vediamo lutti, dovunque sentiamo gemiti. Le città sono state distrutte, le fortezze abbattute, le campagne devastate, la terra è stata ridotta a un deserto. (trad. Gandolfo 1993, con modifiche dell'autrice)

Su questo scenario di declino generale il vescovo allarga il suo sguardo, prima di focalizzarlo sulla sua città e anche alla fine, come già rilevato ${ }^{84}$. Nel dipingerlo, l'autore combina verosimilmente spunti attinti da Ezechiele su città desolate e campi devastati ${ }^{85}$ con l'allusione a un verso virgiliano sulla caduta di Troia, che già Girolamo e Pelagio, ad esempio, hanno ${ }^{\text {citato }}$, riferendosi ai disastri del loro tempo, anch'essi in discorsi di parenesi al distacco dal mondo ${ }^{86}$. Più sottile, però, è il richiamo virgiliano di Gregorio, che per la presenza di gemitus (al posto del virgiliano pauor) potrebbe essere mutuato per il tramite geronimiano e, in più, risulta finemente screziato dalla variatio ubique / undique, incardinata nel perfetto parallelismo ubique - aspicimus, undique - audimus, che gli conferisce un'epica gravità.

Il passo in questione ed altri analoghi attestano la tendenza di molti autori cristiani a risemantizzare il motivo storiografico e letterario del mondo in declino, già diffuso nella latinità pagana, a favore di scelte ascetiche e caritative cristiane. Specialmente Gregorio, infatti, sulla scia di Agostino, punto di riferimento nella sua riflessione teologica, vede nelle catastrofi un mezzo attraverso cui Dio corregge con flagelli i figli che ama (secondo Prou. 3, 12 ed Hebr. 12, 6), per guidarli alla maturità spirituale e alla salvezza ${ }^{87}$. Nella decima e ultima omelia su Ezechiele, infatti, il vescovo, indotto a sospendere la sua predicazione sul profeta dalle angustie presenti, trae tuttavia da esse un motivo di edificazione:

Ipse etenim qui nos creauit etiam pater nobis factus est per adoptionis spiritum quem dedit. Et aliquando filios pane nutrit, aliquando flagello corrigit, quia per dolores et unlnera et munera ad hereditatem perpetuam erudit ${ }^{88}$.

83 Greg. M. in Ezech. 2, 6, 22 (ed. Adriaen, 310, 524-527).

84 Cf. supra, testo e n. 81 .

$85 \mathrm{Ez} .12,20$ : et ciuitates quae nunc habitantur desolatae erunt terraque deserta.

86 Cf. Verg. Aen. 2, 368-369: Crudelis ubique luctus, ubique pauor et plurima mortis imago, rievocato anche in Hier. epist. 60, 16 (ed. Hilberg, CSEL 54, 571, 2-3): ubique luctus, ubique gemitus et plurima mortis imago; epist. 127, 12 (ed. Hilberg, CSEL 56.1, 155, 10 11); Pelag. epist. ad Demetr. (PL 30, 45). In merito cf. Courcelle 1964, 25. 201, n. 1 e 257, n. 7.

87 Cf. Aug. serm. 296 (ed. Morin, Miscellanea Agostiniana 1, 409, 15-18); analogamente Aug.. exc. urb. 3 (ed. O'Reilly, 254, 142-143). Prou. 3, 12 (Hebr. 12, 6) non mi risulta usato da Girolamo come chiave interpretativa delle catastrofi del presente.

88 Greg. M. in Ezech. 2, 10, 24 (ed. Adriaen, 397-398, 624-628). 
Colui che ci ha creati è diventato per noi anche Padre in virtù dello spirito di adozione che ci ha donato. E qualche volta nutre i figli con pane, qualche volta li corregge con la verga, onde prepararli, attraverso dolori e castighi, all'eredità eterna. (trad. Gandolfo 1993, 293)

Eppure - egli rileva amaramente nell'omelia sesta, qui in esame:

finem non habent flagella caelestis iustitiae, quia nec inter flagella correctae sunt actionis culpae ${ }^{89}$.

E i flagelli della giustizia celeste non hanno termine, poiché neppure in mezzo ai flagelli si emendano le colpe. (trad. Gandolfo 1993, 171)

La "colpa" a cui l'autore allude senza meglio specificarla è da individuare, suppongo, in ogni forma di attaccamento alle cose terrene, considerato deviante a livello spirituale e assurdo a livello razionale, visto lo stato deplorevole in cui versa il mondo presente.

Perciò acquistano particolare pregnanza le esortazioni al distacco interiore dalle cose terrene e all'ascesi, che il vescovo indirizza ai suoi ascoltatori, ponendole strategicamente, in una sorta di "Ringkomposition", all'inizio del suo excursus:

Quid est ergo quod in hac uita libeat, fratres mei??0

Cosa ci può dunque attirare in questa vita, fratelli miei? (trad. Gandolfo 1993, 171)

e a suo suggello:

Despiciamus ergo ex toto animo hoc praesens saeculum uel exstinctum; finiamus mundi desideria saltem cum mundi fine ${ }^{91}$.

Disprezziamo dunque con tutta l'anima questo mondo presente come estinto; poniamo fine ai nostri desideri mondani almeno con la fine del mondo. (trad. Gandolfo 1993, 175)

Di questo mondo, che a Gregorio pare avviarsi al suo epilogo, Roma assurge qui a simbolo: ambiguo, perché ne rappresenta all'apice la gloria, il fascino e insieme la loro fallacia e caducità ${ }^{92}$.

89 Ibid., 6, $22(310-311,529-531)$.

90 Ibid. $(311,532-533)$.

91 Ibid., 24 (313, 601-603).

92 In merito mi pare calzante l'osservazione di Demandt 1984, 497: “Die Vergangenheit Roms lehrt die Vergänglichkeit alles Irdischen, nicht nur dass, sondern auch wie es verdirbt, denn die Struktur des Gewebes zeigt sich in der Auflösung”. 


\section{Conclusione}

Questo senso di caducità e di declino emerge nei testi di entrambi gli autori, nei quali è soggetto a un'alta stilizzazione letteraria, intessuta di reminiscenze classiche (più evidenti in Girolamo) e bibliche (più pervasive in Gregorio), tanto che è difficile talora discernere tra tratti realistici e dotte allusioni.

Il tema della caduta di Roma è inquadrato nella loro esegesi a Ezechiele e da ciò riceve un connotato profetico: più tenue in Girolamo, che lo relega sostanzialmente nei prologhi del suo commentario; più marcato in Gregorio, che lo integra nell'esposizione di un capitolo centrale di Ezechiele: Ez. 24 sulla caduta di Gerusalemme, e intesse il suo discorso su un andirivieni tra riflessioni sul presente e profezia biblica, di cui egli rileva il compimento nella realtà da lui vissuta ${ }^{93}$.

Per entrambi, comunque, la crisi di Roma è sineddoche della fine del mondo: così nel loro "Rombild" si riflette la loro "Weltanschauung", nella quale - pur con accentuazioni diverse nei due autori - ogni catastrofe è indizio del giudizio divino, che si esprime anche nella storia, castigando il peccato e richiamando alla conversione, come già hanno rivelato i profeti veterotestamentari.

Peccato e conversione sono da intendere a livello non solo individuale, ma anche collettivo ${ }^{94}$. Sembra, infatti, che i due teologi vedano espiarsi sotto i colpi dei barbari una sorta di peccato "endemico" della civiltà romana, della quale si sentono parte, senza però risparmiarle note critiche ${ }^{95}$. Ecco perché nel loro

93 Si notino, infatti, le formulazioni di raccordo tra un'osservazione sul presente e il detto biblico che la riflette in termini allegorici: completum esse uideamus: Greg. M. in Ezech. 2, 6, 22 (ed. Adriaen, 311, 538); impletum est in ea quod ... per prophetam dicitur: ibid., 23 (312, 577-578) ovvero contigit ei quod de Iudaea nouimus per prophetam dictum: ibid. (312, 588-589); dicatur e dicamus prima di citazioni profetiche: ibid., 22 (311, 558 e 312, 565-566) e ibid., 23 (312, 587).

94 In proposito, infatti, sia Girolamo sia Gregorio usano la prima persona plurale, che non va intesa, a mio avviso, come pluralis maiestatis o humilitatis: cf. ad es. Hier. epist. 60, 16 (ed. Hilberg, CSEL 54, 571, 4): Romanus orbis ruit, et tamen ceruix nostra erecta non flectitur; ibid. 17 (572, 4-6): olim offensum sentimus nec placamus Deum. Nostris peccatis barbari fortes sunt, nostris uitiis Romanus superatur exercitus; epist. 128, 5 (ed. Hilberg, CSEL 56.1, 161, 9-10: v. supra, n. 66); Greg. M. in Ezech. 2, 6, 22 (ed. Adriaen, 310311, 529-531 citato sopra, testo e n. 89) ; epist. 9, 240 (ed. Norberg, CChr.SL 140 A, 824, 37-39): Romana enim ciuitas peccatis nostris facientibus diuersis est attrita languoribus (qui nostris potrebbe intendersi riferito a Gregorio stesso e al suo "entourage"); in euang. 1, 17, 16 (ed. Étaix, 130, 363-365): in questa omelia, rivolta ad altri vescovi, Gregorio intende probabilmente i peccati di un clero negligente; così in epist. 11, 31 a tutti i vescovi della Sicilia (ed. Norberg, CChr.SL 140 A, 919, 5-8). Sugli attacchi barbarici come punizione cf. anche supra, testo e n. 15.

95 Si noti, a titolo indicativo, l'assimilazione di Roma a Babilonia in Hier. epist. 45, 6 e 46, 12 (ed. Hilberg, CSEL 54, 327, $12-13$ e 341, 16-342, 15) e la critica di superbia, 
discorso su Roma trapela una certa ambiguità, tra senso di appartenenza a quell'antica civiltà e presa di distanze dai caratteri di idolatria, rapacità e superbia, che essi le associano.

In effetti, le esternazioni di Girolamo sulla caduta di Roma vanno collocate sullo sfondo della polemica coi pagani sulle cause di crisi dell'impero. Questa, tuttavia, nei testi qui considerati rimane in secondo piano rispetto alla parenesi ascetica che egli rivolge alle sue interlocutrici, esponenti di un ceto senatorio convertitosi solo in parte al Cristianesimo, con la conseguenza di forti tensioni etiche ed ideologiche al proprio interno.

Mutata è la realtà socio-religiosa in cui vive Gregorio; in lui manca, quindi, l'istanza di un'apologetica anti-pagana, ma più accentuato, concreto e inesorabile è il presagio della fine del mondo, poiché a suscitarlo sono non solo le distruzioni apportate dai barbari, ma anche le numerose calamità naturali e il degrado crescente nel tessuto urbano della Roma imperiale. D'altronde a quell'epoca si è ormai dissolto l'impero d'Occidente, che ai tempi di Girolamo ancora sussisteva, benché indebolito.

Nel clima di incertezza e inquietudine sociale connesso a questi scenari politici, si potrebbe supporre che la proposta ascetica incorniciante il "Rombild" catastrofico di Girolamo e di Gregorio ${ }^{96}$ si profili per loro e i loro destinatari come una salus uictis ${ }^{97}$, ovvero come unica possibile fonte di nuove certezze trascendenti, non terrene, nutrite dalla fede cristiana, non più garantite dall'assetto politico romano - e al contempo come risorsa per far fronte costruttivamente, col distacco dai propri beni e il loro impiego a scopi caritativi, alle miserie materiali e sociali del mondo antico irreversibilmente in declino.

\section{Bibliografia}

Arnaldi, Girolamo (1982), "Rinascita, fine, reincarnazione e successive metamorfosi del senato romano (secoli V-XII)", in: Archivio della Società Romana di Storia Patria $105,5-56$.

Bartelink, Gerard J. M. (2000), "The Theme of the End of the World in the Works of Gregory the Great", in: Caroline Kroon/Daniël den Hengst (eds.), Ultima Aetas. Time, Tense and Transience in the Ancient World. Studies in Honour of Jan den Boeft, Amsterdam, 139-146.

Boesch Gajano, Sofia (2004), Gregorio Magno. Alle origini del Medioevo (sacro/santo, nuova serie 8), Roma.

immoderatum gaudium e di rapacità in Greg. M. in Ezech: 2, 6, 23 (ed. Adriaen, $312-$ 313, 574-576. 580-581.586-587. 596-597, citati supra, testo e n. 68.72. 75. 77).

96 Proposta più evidente nelle lettere di Girolamo che nel suo commentario a Ezechiele, in cui essa rimane semmai sullo sfondo, implicata dalla natura stessa del dialogo tra l'autore, maestro spirituale, e la dedicataria, sua discepola.

97 Verg. Aen. 2, 354. 
Consolino, Franca E. (2009), "Priamo, Pompeo e Giovanni Battista. Caratteri e limiti dell'allusività in Giovenco 3,33-72", in: Henriette Harich-Schwarzbauer/Petra Schierl (eds.), Lateinische Poesie der Spätantike, Basel, 159-177.

Courcelle, Pierre (1964), Histoire littéraire des grandes invasions germaniques, Paris, $3^{\mathrm{e}}$ éd.

Demandt, Alexander (1978), Metaphern für Geschichte. Sprachbilder und Gleichnisse im historisch-politischen Denken, München.

Demandt, Alexander (1984), Der Fall Roms. Die Auflösung des römischen Reiches im Urteil der Nachwelt, München.

Doignon, Jean (1990), “Oracles, prophéties, 'on-dit' sur la chute de Rome (395-410). Les réactions de Jérôme et d'Augustin”, in: Revue des Études Augustiniennes 36, 120 146.

Feichtinger, Barbara (1995), Apostolae apostolorum. Frauenaskese als Befreiung und Zwang bei Hieronymus, Frankfurt am Main.

Feichtinger, Barbara (1998), "Glaube versus Aberglaube. Der Untergang Roms in den Augen der Zeitgenossen", in: Ernst Dassmann/Klaus Thraede/Josef Engemann (eds.), Chartulae. Festschrift für Wolfgang Speyer (Jahrbuch für Antike und Christentum. Ergänzungsband 28), Münster, 145-166.

Freund, Stefan (2009), Laktanz. Divinae Institutiones Buch 7: De vita beata, Einleitung, Text, Übersetzung, Kommentar, Berlin.

Fürst, Alfons (2003), Hieronymus. Askese und Wissenschaft in der Spätantike, Freiburg im Breisgau.

Ghilardi, Massimiliano/Pilara, Gianluca (eds.) (2010), I Barbari che presero Roma. Il sacco del 410 e le sue conseguenze, Roma.

Gregorovius, Ferdinand (1988), Geschichte der Stadt Rom im Mittelalter, 1. 1, 2. Auflage. Herausgegeben von Waldemar Kampf, München.

Hagendahl, Harald (1958), The Latin Fathers and the Classics, Göteborg.

Häussler, Ralph (1964), "Vom Ursprung und Wandel des Lebensaltervergleichs", in: Hermes 92, 313-341.

Heather, Peter J. (2005), The Fall of the Roman Empire, London.

Inglebert, Hervé (1996), Les Romains chrétiens face à l'histoire de Rome: histoire, christianisme et romanités en Occident dans l'Antiquité tardive (IIIe-Ve siècles), Paris.

Kessler, Stephan Ch. (1995), Gregor der Große als Exeget. Eine theologische Interpretation der Ezechielhomilien (Innsbrucker Theologische Studien, 43), Innsbruck/Wien.

Krautheimer, Richard ( $\left.{ }^{2} 1996\right)$, Rom. Schicksal einer Stadt 312-1308, München.

Leclercq, Jean (1963), Otia monastica. Etudes sur le vocabulaire de la contemplation au Moyen Âge (Studia Anselmiana 51), Roma.

Luiselli, Bruno (1998), "La cultura latina dei secoli IV-VI di fronte ai barbari invasori dell'impero", in: Marcello Rotili (ed.), Incontri di popoli e culture tra Ve IX secolo (Atti delle V giornate di studio sull'età romano-barbarica), Napoli, 19-30.

Luiselli, Bruno (1992), Storia culturale dei rapporti tra mondo romano e mondo germanico, Roma.

Luneau, Auguste (1964), L'histoire du salut chez les Pères de l'Église. La doctrine des âges du monde, Paris.

Manselli, Raoul (1983), Art. "Gregor V (Gregor der Große)", in: Reallexikon für Antike und Christentum 12, Stuttgart, 930-951.

Markus, Robert A. (1997), Gregory the Great and His World, Cambridge.

Mohrmann, Christine (1977), "Episkopos-Speculator”, in: Ead. (ed.), Etudes sur le latin des Chrétiens 4, Roma, 231-252.

Müller, Barbara (2009), Führung im Denken und Handeln Gregors des Grossen (Studien und Texte zu Antike und Christentum 57), Tübingen. 
Paschoud, François (1967), Roma aeterna. Etudes sur le patriotisme Romain dans l'occident Latin à l'époque des grandes invasions, Rome.

Pietri, Charles (1991), "La Rome de Grégoire", in: Gregorio Magno e il suo tempo, 1 (Studia Ephemeridis Augustinianum 33), Roma, 9-32.

PLRE: The Prosopography of the Later Roman Empire, 1-3, ed. A. H. M. Jones, J. R. Martindale, J. Morris, Cambridge 1971-1992.

Pohlmann, Karl F. (2008), Ezechiel. Der Stand der theologischen Diskussion, Darmstadt.

Rebenich, Stefan (1992), Hieronymus und sein Kreis: prosopographische und sozialgeschichtliche Untersuchungen, Stuttgart.

Rebenich, Stefan (2002), Jerome (The early Church Fathers), London.

Rebenich, Stefan (2009), "Christian Ascetism and Barbarian Incursion: The Making of a Christian Catastrophe", in: Journal of Late Antiquity 2, 49-59.

Ricci, Cristina (2002), Mysterium Dispensationis. Tracce di una teologia della storia in Gregorio Magno (Studia Anselmiana 135), Roma.

Ricci, Cristina (2003), "La profezia in Gregorio Magno", in: Augustinianum 43, 125 200.

Santi, Francesco (2008), Art. "Homiliae in Hiezechielem”, in: Giuseppe Cremascoli/ Antonella Degl'Innocenti (eds.), Enciclopedia gregoriana, 163-166.

Schmidt, Roderich (1956), "Aetates mundi. Die Weltalter als Gliederungsprinzip der Geschichte", in: Zeitschrift für Kirchengeschichte 67, 288-317.

Schwank, Hans (1934), Gregor der Große als Prediger, Hannover.

Siniscalco, Paolo (1983), "Roma e le concezioni cristiane del tempo e della storia nei primi secoli della nostra era", in: Roma, Costantinopoli, Mosca, Atti del I Seminario Internazionale di Studi Storici "Da Roma alla terza Roma" (21-23 aprile 1981), Roma, 31-62.

Stemberger, Günter (1987), Juden und Christen im Heiligen Land, München.

Sugano, Karin (1983), Das Rombild des Hieronymus, Frankfurt am Main/Bern.

Vogt, Ernst (1981), Untersuchungen zum Buch Ezechiel, Roma.

Zocca, Elena (1995), "La senectus mundi. Significato, fonti e fortuna di un tema ciprianeo", in: Manlio Simonetti/Paolo Siniscalco (eds.), Studi sul Cristianesimo antico e moderno in onore di Maria Grazia Mara, Augustinianum 35, 641-677.

Zwierlein, Otto (1978/2004), "Der Fall Roms im Spiegel der Kirchenväter", in: Zeitschrift für Papyrologie und Epigraphik 32, 45-80 / Lucubrationes Philologae, Bd. 2: Antike und Mittelalter, Berlin/New York, 427-466. 